\title{
LoRa Alliance Certification
}

\author{
Derek Hunt
}

Technical Fellow, Semtech, and LoRa Alliance ${ }^{\circledR}$ Technical Committee Vice-Chair, Fremont, CA, United States

E-mail: derek.hunt@lora-alliance.com

Received 04 March 2020; Accepted 27 May 2020;

Publication 30 April 2021

\begin{abstract}
LoRaWAN ${ }^{\circledR}$ Certification of devices is critical for effective and efficient mass deployment of LoRaWAN Networks, as it ensures devices will work on any network, under all conditions. Certified Devices significantly reduce the support costs as any product failures detected later when the device is deployed is far more expensive to repair and poor RF performance of the device increase number of gateways needed and cost of the network infostructure.
\end{abstract}

Keywords: LoRa alliance authorised test houses (ATH), industrial, scientific and medical radio band (ISM band), low power, wide area (LPWA) networking protocol (LoRaWAN ${ }^{\circledR}$ ), network server (NS), radio frequency (RF), test control layer (TCL).

\section{Introduction}

It is essential for any successful network to have devices that correctly communicate with it, and work as expected. This is even more important in a low cost network where every fault or incident will cost time and money to rectify. It is critical that devices are LoRaWAN Certificated to ensure that

Journal of ICT, Vol. 9_1, 13-20. River Publishers

doi: $10.13052 /$ jicts2245-800X.912

This is an Open Access publication. (C) 2021 the Author(s). All rights reserved. 
in a LoRaWAN ${ }^{\circledR}$ Network they interoperate with the Gateways and Network Servers and guarantee that all LoRaWAN end-devices are able to work under any network condition whilst in any public or private network. There are over 350 certification tests successfully passed (as of end of 2020), for region in Europe, North America, India, Korea and Asia.

There is no formal LoRa Alliance Certification tests for Network Servers at present, instead vendors perform interoperability tests for roaming and activation interfaces based on the LoRaWAN Backend Interfaces Specifications.

\section{LoRaWAN Certification}

For the LoRaWAN technology to succeed is essential that device manufacturers can provide very low cost devices that are fully interoperable and functional. With this in mind the LoRaWAN Certification program was designed to test all of the protocol defined in the LoRaWAN link layer Specification [1] in cost effective manner, In many technologies, testing is so expensive that only modules are tested, and designs have to use certified modules. With LoRaWAN, both modules and chip-down designs are possible, which optimizes costs. For manufactures and testing facilities it is important that there is not a need for very expensive test equipment. To achieves these requirements the Certification protocol test were designed and specified so that they can be tested without any expensive test equipment using only a standard LoRaWAN Gateway and a test harness running on a PC as the Network Server (NS) and the Test Control Layer (TCL).

As well as very minimal equipment expenditures the other key component determining the cost of certification is the time taken to execute the tests. The tests are defined so that they can be executed in a fully automatic test harness that keeps the test time to a minimum. The intention is to keep the cost of Certification competitive and so that the testing can be completed within 1 day, not weeks or months as it is with other technologies.

\subsection{Certification Test Set Up}

The Device Under Test (DUT) and the LoRaWAN Gateway (GW) are housed in a shielded box to avoid any other radio transmission in the ISM band that may affect the test results. Also as the end-devices are likely to be small radio devices the certification has been designed to only use the LoRaWAN Radio connection on the device (DUT) to be certified and does not require any additional test connections. 


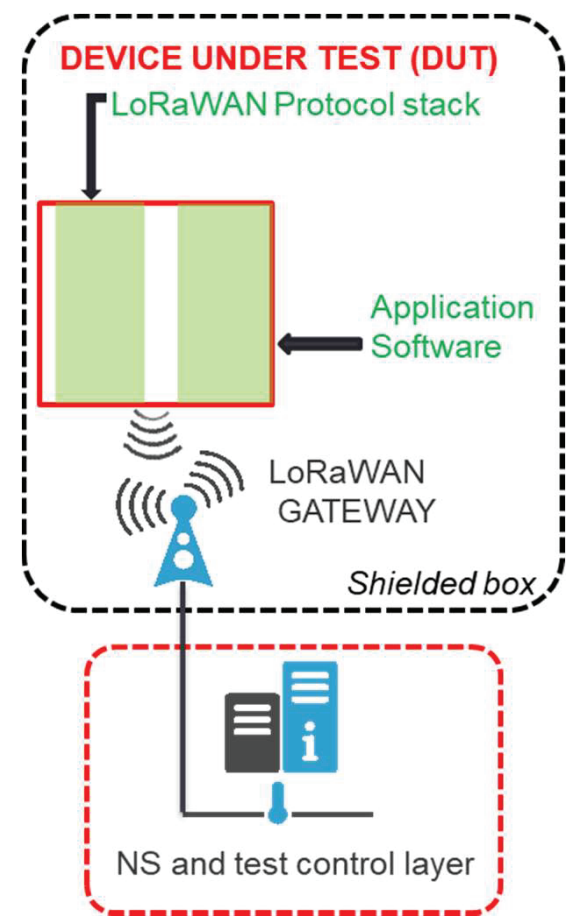

Figure 1 Typical certification test infrastructure.

\subsection{Certification Test Procedure}

The LoRa Alliance Certification Committee has full responsibility for the creation a comprehensive test procedure for DUT that will ensure the correct LoRaWAN behaviour of end devices and includes testing all of the MAC commands as well as functional testing of the device.

The main function of the Gateway is to forward LoRaWAN packets from the DUT and send them to the Network Server and hence it has very little involvement in the LoRaWAN protocol, so the protocol tests are between the End Device (DUT) and the Network Server (NS).

The Certification process sends and receives over 1000 messages between the NS and DUT and as LoRaWAN operates in the Sub 1GHz ISM bands. The band has slightly different requirements in the various regions around the world and the LoRa Alliance has produced a LoRaWAN Regional Parameters [2] document to specify the use of LoRaWAN in these different regions. The LoRaWAN certification has different certification test requirements 
to reflect these different regions therefore, LoRa Alliance Certification is granted on a per region basis.

The LoRa Alliance Certification program does not test the regulatory requirements of the ISM band for the country or region it is expected to operate in, but the devices must separately pass all of these regulatory requirements that are required for the country or region it is deployed in, currently there are around 60 countries that have granted regulatory approval to LoRaWAN Devices. Typically the Regulatory tests check that the parameters of a device are below the maximum permitted for that region and would consist of tests like maximum output power measurements, spurious emissions tests etc. The LoRaWAN Certification tests for adherence to the LoRaWAN Protocol and the actual output power which for some devices can be significantly less the max permitted power.

A full list of the Certification Test Specification can be found in the resources tab on the Certification Pages on the LoRa Alliance website: https://lora-alliance.org/lorawan-certification.

\subsection{LoRa Alliance Certification Authorised Test Houses}

The LoRa Alliance governs and operates the LoRaWAN Certification program and has several appointed Authorised Test Houses (ATH) to do the Certification testing. These ATHs have worldwide location to do the Certification. Each physical location of these ATH's are audited and authorised by the LoRa Alliance for each LoRaWAN Version [1] and each LoRaWAN Region [2]. The alliance issues Certificates of Authorisation (CoA) to each Test House stating what they are authorised to certify. As devices are needed to be tested against different regions the aim is that the these ATHs can provide testing at locations near the device developer even if the device is to be certified for a different region i.e. a device may be designed in Europe and tested in a European ATH against the North American LoRaWAN Region specification [2]. The list of available ATHs can be found at https: //lora-alliance.org/certification-test-houses.

\subsection{LoRa Alliance Certification Certificate}

When a device is successfully certified by the one of the ATH's the results are sent to the LoRa Alliance and the LoRa Alliance issues the Certificate to the manufacture and shows the device in the master list of certified products. This list of Certified products is displayed on the LoRaWAN Showcase on the LoRa Alliance Website. https://lora-alliance.org/showcase. 


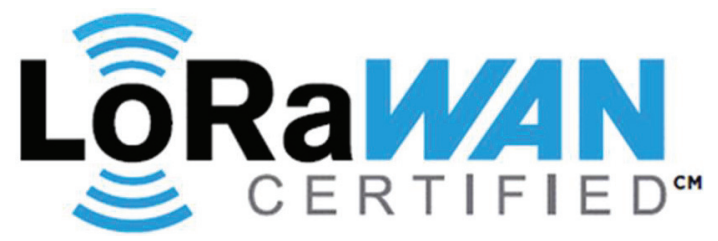

Figure 2 LoRaWAN certified ${ }^{\mathrm{CM}}$ logo.

\subsection{LoRa Alliance Certification Mark}

Devices that pass the LoRaWAN Certification are permitted to display the LoRaWAN Certified ${ }^{\mathrm{CM}}$ Logo on the product and device literature

\subsection{LoRa Alliance Pre-Certification Test Tool}

To allow the device manufacturer to pre-test the LoRaWAN protocol of their device before sending it to an ATH the LoRa Alliance has commissioned and made available free to all LoRa Alliance Members a LoRaWAN Certification Test Tool (LCTT). https://lora-alliance.org/lorawan-certification. This tool is intended to be used at device designers own premises and it is downloaded from the LoRa Alliance Web Site to a local PC which is used to run the Network Server and the Test control Layer which is connected to a local LoRaWAN Gateway. The LCTT does not give certification nor the use of the certification logo, but it ensures that the device is ready to be certificated at the one of the ATHs and is provides significant assistance in developing certified products. The LCTT also has a debug mode that allows detailed investigation in any faults found while developing the device and can also be used for regression testing after any change to the device has been made

The Device to be tested is shown as the DUT see Figure 3.

\subsection{LoRa Alliance RF Performance Guidelines for a Device}

The LoRaWAN Certification tests the protocol to prove the adherence to the LoRaWAN link layer Specification [1] but another area that is critical to the usability and reliability of a device is the RF Performance. The LoRa Alliance Certification Committee has produced a comprehensive set of guidelines on how to measure the RF Performance of the device to measure the device's output power at various frequencies and measure the device's sensitivity at various frequencies. Generally the receivers sensitivity performance is aligned with the transmit performance of the device but this is not always 


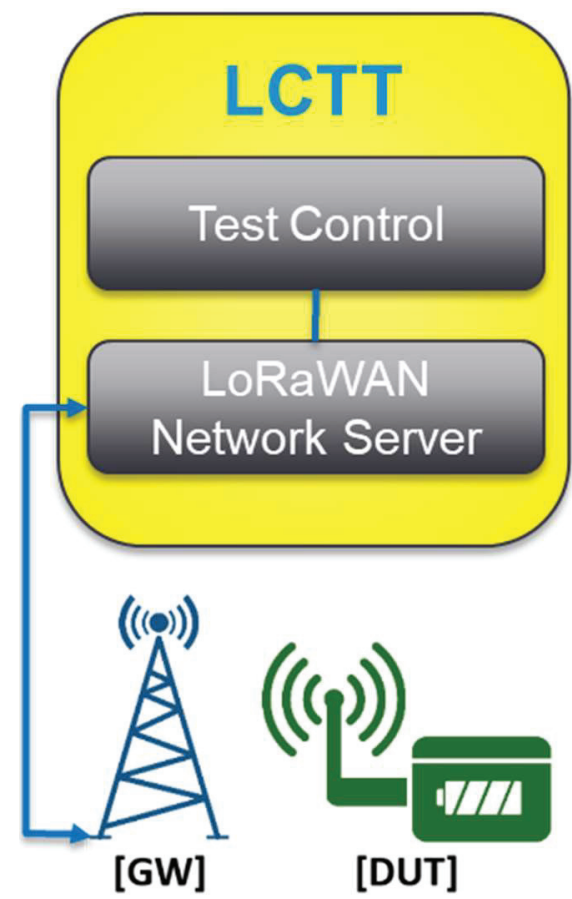

Figure 3 LoRaWAN certification test tool infrastructure.

the case These guidelines can be used by device manufactures to check the performance of their device prior to sending it to an ATH for the official measurement. There is no pass or fail on the RF performance measurements as the application and physical size of each device and the way it is intended to be used could mean that even a device with a low output power is suitable for some application and hence it is not necessary for it to have a greater output power as this will just drive more cost in to the device when it is not needed. The output power and sensitivity measurements of the device are used by the network operator to ensure it will have reliable communication with the device wherever it is installed in their network.

\section{Conclusion}

For a LoRaWAN device to interoperate successfully in a LoRaWAN network or roam over multiple networks it is essential that it has passed the LoRa Alliance Certification tests that are relevant for the version of LoRaWAN link 
layer Specification [1] and the LoRaWAN Region Parameters [2] used for the device. It is also essential that its RF Performance is measured so that when it is operated in a LoRaWAN networks it can be guaranteed to work under all network conditions.

\section{Acknowledgements}

LoRa Alliance Certification Committee.

\section{References}

[1] LoRaWAN L2 Specification

[2] LoRaWAN Regional Parameters Specification

\section{Biography}

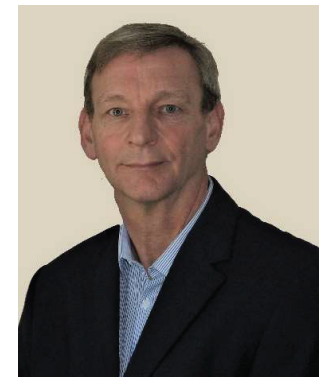

Derek Hunt is a Director of Certification for LoRa Alliance and Certification Committee Chair. He has been working on LoRa Systems and specifications since 2014, at Semtech and Actility prior to become part of the LoRa Alliance.

Before working on LoRa Derek has had over 30 years telecommunications experience working in different hardware, software and ASIC development and management roles as well as consultant roles for Ericsson, Marconi and GEC-Plessey Telecommunication at locations in Europe and North America. 
